\title{
Enriquecimento ambiental aplicado a tamanduás-mirins (Tamandua tetradactyla) no Aquário de São Paulo: estudo de caso
}

\author{
Fernanda Buck de Godoy Peixoto ${ }^{1}$, Maryana Tursi Gonçalves Ambrózio ${ }^{1}$, Helen \\ Colbachini $^{2}$ (D), Fabiana Lúcia André Padilha ${ }^{2}$ (D) \& Fabiana Rodrigues Costa $^{3}$ (D)
}

(1) Universidade Federal de São Paulo - campus Diadema, Instituto de Ciências Ambientais, Químicas e Farmacêuticas, Departamento de Ciências Biológicas, Rua Prof. Artur Riedel 275, Jardim Eldorado, Diadema 09972-270, São Paulo, Brasil.

(2) Aquário de São Paulo, Rua Huet Bacelar 407, Ipiranga 04275-000, São Paulo, Brasil. E-mail: helen@aquariodesaopaulo.com.br

(3) Universidade Federal do ABC - campus São Bernardo do Campo, Centro de Ciências Naturais e Humanas, Laboratório de Paleontologia de Vertebrados e Comportamento Animal, Rua São Paulo s/n, Jardim Antares, São Bernardo do Campo 09606-070, São Paulo, Brasil. E-mail: fabiana.costa@ufabc.edu.br

Peixoto F.B.G., Ambrózio M.T.G., Colbachini H., Padilha F.L.A. \& Costa F.R. (2019) Enriquecimento ambiental aplicado a tamanduás-mirins (Tamandua tetradactyla) no Aquário de São Paulo: estudo de caso. Pesquisa e Ensino em Ciências Exatas e da Natureza, 3(2): 119-124.

http://dx.doi.org/10.29215/pecen.v3i2.1256

Editor acadêmico: Manoel dos Santos Filho. Recebido: 16 Abril 2019. Aceito: 25 Junho 2019. Publicado: 02 Outubro 2019.

Resumo: Objetivou-se aqui aplicar diferentes enriquecimentos ambientais a dois indivíduos de tamanduámirim (Tamandua tetradactyla) do Aquário de São Paulo em três fases: (1) pré-enriquecimento; (2) enriquecimento; (3) pós-enriquecimento. Alterações nas frequências de alguns comportamentos interativos e associados à locomoção foram observadas para ambos os indivíduos, de forma a apontar que a aplicação dos enriquecimentos teria favorecido nos animais maior atividade, promovendo estímulos e refletindo, portanto, maior bem-estar, além de contribuir para o aumento da variabilidade e complexidade do ambiente onde estes são mantidos.

Palavras chave: Comportamento Animal, Etologia, Xenarthra, Vermilingua.

\section{Environmental enrichment applied to southern tamandua (Tamandua tetradactyla) at Aquário de São} Paulo: a case study

Abstract: The aim of this study was to apply different environmental enrichment to two subjects of southern tamandua (Tamandua tetradactyla) from Aquário de São Paulo in (1) pre-enrichment; (2) enrichment; and (3) post-enrichment phases. Changes in the frequencies of some interactive behaviors associated to locomotion were reported for both subjects, which indicate that the application of enrichment would have favored higher levels of activity by promoting stimuli and, thus, greater well-being, besides contributing to the increase on the variability and complexity of the environment where they are maintained.

Key words: Animal behavior, Ethology, Xenarthra, Vermilingua.

O uso do enriquecimento ambiental em zoológicos é muito importante por propiciar um maior bem-estar aos animais, aproximando-os de seu comportamento natural (Dias et al. 2010). Este tipo de técnica pode ser aplicada a diferentes grupos animais, vertebrados e invertebrados (e.g., Carducci \& Jakob 2000; Hurme et al. 2003), uma vez em cativeiro (zoológicos, refúgios, 
granjas e laboratório) (e.g., Maple 2007), sendo usada inclusive para xenartros (superordem que abarca os tamanduás, tatus e preguiças) (Vizcaíno \& Loughry 2008). Dentre estes, os tamanduásmirins (Tamandua tetradactyla Linnaeus, 1758) são representados por animais solitários pouco estudados quando em vida livre e com ocorrência relativamente comum em zoológicos brasileiros (Catapani 2014). Em relação a esta espécie, existem ainda muitas lacunas de conhecimento no que concerne a seus aspectos ecológicos e comportamentais (Redford 1994), e grande parte das informações sobre a biologia da espécie advém de estudos com animais em cativeiro, uma vez que sua observação em ambiente natural é bastante difícil (Superina 2012). Catapani (2014), por exemplo, investigou o efeito do convívio com coespecíficos sobre o comportamento exibido por tamanduás-mirins. Outros destes aspectos, como reprodução (Silveira 1968; Eisenberg \& Redford 1999; Rodrigues \& Marinho-Filho 2008), interações (Medri et al. 2003) e padrão de atividade (Lubin 1983; Montgomery 1985; Rodrigues \& Marinho-Filho 2003) já foram abordados, não obstante muito deste conhecimento ainda precise ser melhor elucidado para a espécie em questão.

O presente estudo (CEUA/UNIFESP, $\mathrm{n}^{0}$ 2013040817) foi realizado com dois indivíduos de tamanduás-mirins adultos (um macho - "Jorge", e uma fêmea - "Lipe") mantidos em recinto no Aquário de São Paulo que segue as especificações da Instrução Normativa $\mathrm{n}^{\circ} 07$ de 30 de abril de 2015 do IBAMA - Instituto Brasileiro do Meio Ambiente e dos Recursos Naturais Renováveis, com substrato de terra e elementos como troncos, galhos, plantas e um "lago" artificial. O estudo foi dividido em três fases: (1) pré-enriquecimento (observação e habituação dos observadores aos comportamentos expressos pelos animais antes da inserção dos enriquecimentos; duração total de 7h30); (2) enriquecimento (observação e registro do comportamento dos animais durante a aplicação dos enriquecimentos; duração total de 10h); e (3) pós-enriquecimento (observação e registro do comportamento dos animais após a retirada dos enriquecimentos; duração de 10h), totalizando $27 \mathrm{~h} 30$ de observação. As análises estatísticas consideraram as fases 2 e 3 . Para fins de controle para avaliação a posteriori da eficácia da aplicação dos enriquecimentos na rotina dos indivíduos, determinou-se, simultaneamente à habituação aos comportamentos exibidos, a frequência dos mesmos na fase 1.

As observações dos animais foram realizadas duas vezes por semana durante $1 \mathrm{~h} 15$ por sessão de observação, no período de 02 de agosto a 03 de novembro de 2016, totalizando sete, oito e oito dias, correspondentes às fases 1,2 e 3, respectivamente. No total foram 23 dias distribuídos no período de 02 a 31 de agosto (fase 1), 01 a 29 de setembro (fase 2) e 05 de outubro a 03 de novembro (fase 3 ), conforme determinado pela rotina do Aquário. $\mathrm{O}$ método aplicado às observações foi o animal focal (Altmann 1974) com registro contínuo. Cada indivíduo foi observado intercaladamente durante $1 \mathrm{~min}$. Ao término das observações, os comportamentos mais relevantes observados na fase 1 foram selecionados para terem suas frequências comparadas entre os períodos com enriquecimento (2) e sem enriquecimento (3). A fase 1 foi utilizada para definição de etograma e habituação dos animais com as observadoras e comparada com a frequência de comportamentos exibidos nas fases subsequentes. Os 23 comportamentos observados seguiram Catapani (2014), com quatro modificações: "alimentar-se" condiz à condição de ingestão de qualquer alimento oferecido aos animais no recinto, compilando as condições alimentar-se de insetos e alimentar-se de papa (i.e., dieta); "lamber pata" foi assim particularizado a partir dos comportamentos lamber-se/limpar-se e lamber-se excessivamente; "andar e farejar" refere-se aos momentos em que o animal esteve simultaneamente andando e farejando o recinto; e "interagir com o outro" foi caracterizado como uma compilação de subir, lamber e seguir o outro indivíduo.

Foram utilizados oito diferentes enriquecimentos (categorias alimentar, social, cognitivo, sensorial e estrutural), sendo estes: comedouros suspensos (alimentar); comedouros labirinto (alimentar/cognitivo); dieta congelada (alimentar); "espelho" (social); chá de erva doce com canela borrifado pelo recinto e vinagre (sensitivos/cognitivos) para estímulo dos sentidos; rede de balanço do recinto (estrutural); e mudança de layout dos elementos então existentes no recinto (estrutural). Cada enriquecimento foi introduzido/realizado em um dia de observação, 
sendo mantidos no recinto dos animais por cerca de 1h (Figura 1). Ao término deste tempo, os enriquecimentos foram retirados do recinto.

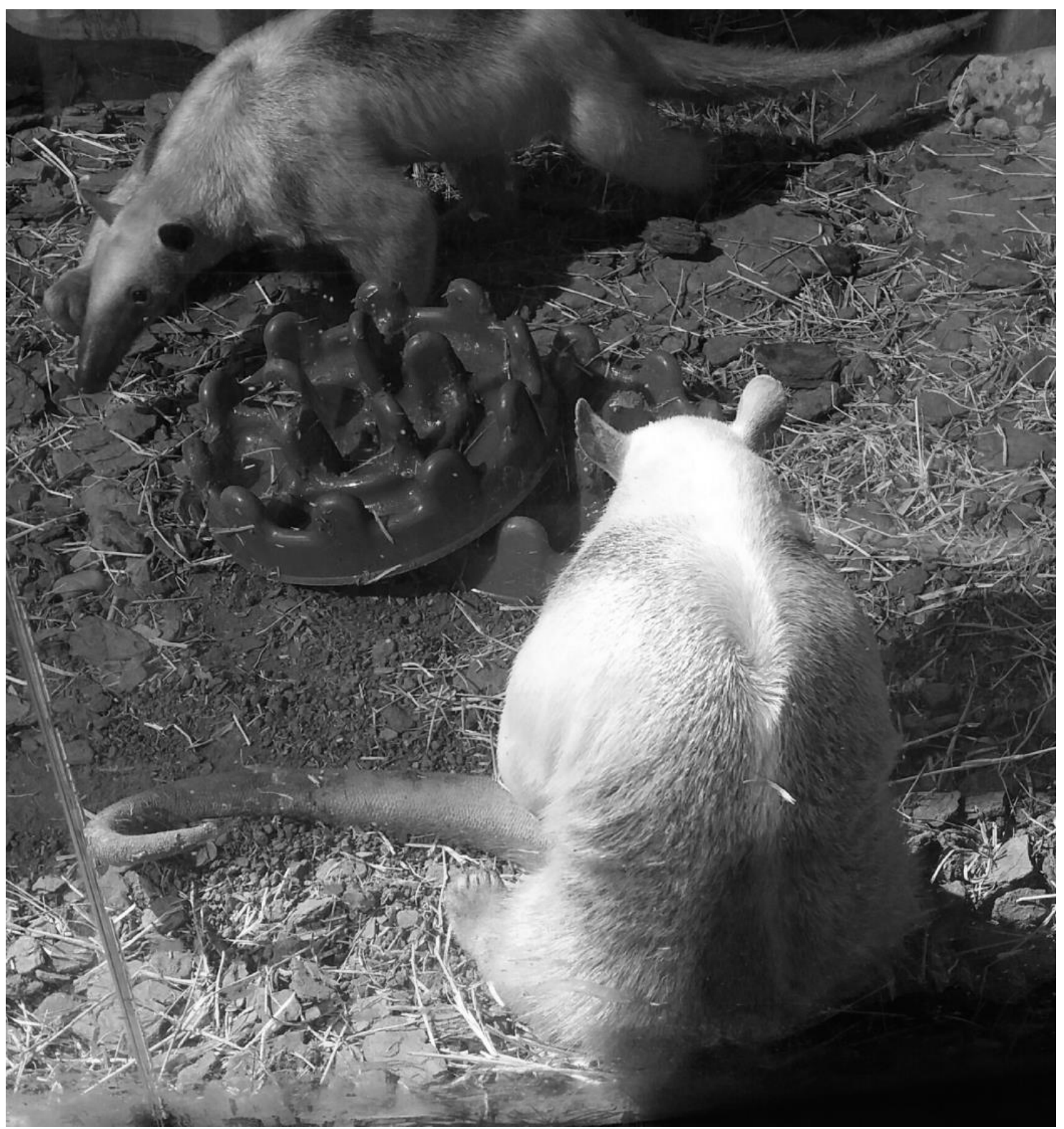

Figura 1. Enriquecimento ambiental denominado "comedouro labirinto": tamanduá macho (ao fundo) e, em primeiro plano, tamanduá fêmea interagindo com o elemento introduzido no recinto. Foto: Maryana Tursi.

Durante este estudo dois novos comportamentos foram observados: "manipular enriquecimento", condizendo à manipulação específica dos elementos do enriquecimento, e "interação com tratadores", consistindo na interação tátil dos animais com os tratadores que eventualmente adentravam o recinto durante as observações. Somando-se estes comportamentos com os 23 retirados de Catapani (2014), totalizaram-se 25 comportamentos observados, dentre os quais foram selecionados os comportamentos mais executados pelos indivíduos: nove da fêmea (Tabela 1) e seis do macho (Tabela 2), e sobre os quais foram aplicados o teste t-Student.

As diferenças entre as frequências dos comportamentos com e sem a aplicação da rotina de enriquecimentos não foi significativa (teste t-Student; Tabelas 1 e 2). No entanto, ainda que tais resultados estatísticos não mostrem a efetividade dos enriquecimentos ambientais como um todo, é possível observar o aumento/redução nas frequências dos comportamentos registrados como mais executados ou considerados como mais relevantes (Tabelas 1 e 2 ). 
Os comportamentos com maior frequência ( $\mathrm{f} \geq 15 \%$ ) durante a fase 1 foram "ficar parado" (15\%), "farejar" (21.10\%) e "andar e farejar" (15.20\%) para a fêmea, e "dormir" (19.30\%), "descansar" (15\%) e "andar e farejar" (37.20\%) para o macho. Comparando-se com as frequências dos comportamentos elencadas nas Tabelas 1 e 2 para as fases 2 e 3, observa-se, em geral, que os enriquecimentos alimentares promoveram maiores frequências de interações para ambos os indivíduos, com destaque para o comedouro labirinto. Além deste último, destaca-se ainda a colocação de rede no recinto, o que teria motivado um maior grau de atividade, principalmente da fêmea (Tabela 1). Os comportamentos que apresentaram maiores frequências durante a fase com enriquecimento, para a fêmea, foram: "deslocar-se", "farejar", "andar e farejar" (locomoção), e "brincar com o outro" (intervenções sociais afiliativas); e para o macho: "brincar com o outro" e "interagir com o outro" (intervenções sociais afiliativas). Compreende-se, portanto, que a introdução dos comportamentos teria promovido o aumento nas relações interativas entre os indivíduos, e uma vez sabendo-se que o estímulo a comportamentos que aumentem os níveis de interação social e, consequentemente, os índices de atividade dos indivíduos, é considerado positivo (Borges et al. 2011), avalia-se como benéfica e, portanto, positiva, a introdução dos enriquecimentos na rotina destes indivíduos.

Nos comportamentos dualísticos "brincar com o outro" e "interagir com o outro", a fêmea apresentou maior frequência em sua execução, o que subentende a maior frequência na intenção de iniciá-los com ou sem enriquecimento. A frequência das interações entre ambos aumentaram após a aplicação dos enriquecimentos (Tabelas 1 e 2), o que mostra que estes teriam tornado os animais mais ativos em relação à expressão de interações mútuas. Uma vez que nenhuma destas interações caracterizou-se como agonística, o que poderia ser consequência do estresse causado pela aplicação dos enriquecimentos, este resultado pode ser interpretado como positivo para os animais em questão (Hashimoto 2008).

Tabela 1. Resultados da aplicação de rotina de enriquecimento para a fêmea obtidos por meio de teste tStudent para os comportamentos de maior frequência elencados.

\begin{tabular}{|c|c|c|c|c|c|c|}
\hline \multicolumn{7}{|c|}{ Resultados da aplicação da rotina de enriquecimento para a fêmea } \\
\hline Comportamentos & $\begin{array}{l}\text { Frequência com } \\
\text { enriquecimento }\end{array}$ & $\begin{array}{c}\text { Média com } \\
\text { enriquecimento }\end{array}$ & $\begin{array}{l}\text { Frequência sem } \\
\text { enriquecimento }\end{array}$ & $\begin{array}{c}\text { Média sem } \\
\text { enriquecimento }\end{array}$ & $\begin{array}{l}\text { Valor de } \\
t \text { (Stat t) }\end{array}$ & $\begin{array}{l}\text { Valor de P } \\
\text { bi-caudal }\end{array}$ \\
\hline Lamber pata & 38 & $4.75 \pm 4.43$ & 53 & $6.62 \pm 7.96$ & -0.58 & 0.56 \\
\hline Ficar parada & 05 & $0.62 \pm 1.06$ & 11 & $1.37 \pm 1.68$ & -1.06 & 0.30 \\
\hline Deslocar-se & 08 & $1 \pm 1.19$ & 04 & $0.5 \pm 0.53$ & 1.08 & 0.29 \\
\hline Escalar & 49 & $6.12 \pm 4.12$ & 54 & $6.75 \pm 4.97$ & -0.27 & 0.78 \\
\hline Farejar & 88 & $11 \pm 5.52$ & 54 & $6.75 \pm 4.97$ & 1.61 & 0.12 \\
\hline Alimentar-se & 53 & $6.62 \pm 6.75$ & 65 & $8.12 \pm 6.57$ & -0.44 & 0.65 \\
\hline Andar e farejar & 59 & $7.37 \pm 6.04$ & 58 & $7.25 \pm 3.37$ & 0.05 & 0.95 \\
\hline Interagir com outro & 08 & $1 \pm 1.06$ & 16 & $2 \pm 2.50$ & -1.03 & 0.31 \\
\hline Brincar com outro & 17 & $2.12 \pm 6.01$ & 05 & $0.62 \pm 1.76$ & 0.67 & 0.50 \\
\hline
\end{tabular}

Tabela 2. Resultados da aplicação de rotina de enriquecimento para o macho obtidos por meio de teste tStudent para os comportamentos de maior frequência elencados.

\begin{tabular}{lcccccc}
\hline \multicolumn{7}{c}{ Resultados da aplicação da rotina de enriquecimento para o macho } \\
\hline Comportamentos & $\begin{array}{c}\text { Frequência com } \\
\text { enriquecimento }\end{array}$ & $\begin{array}{c}\text { Média com } \\
\text { enriquecimento }\end{array}$ & $\begin{array}{c}\text { Frequência sem } \\
\text { enriquecimento }\end{array}$ & $\begin{array}{c}\text { Média sem } \\
\text { enriquecimento }\end{array}$ & $\begin{array}{c}\text { Valor de } \\
\text { t (Stat t) }\end{array}$ & $\begin{array}{c}\text { Valor de P } \\
\text { bi-caudal }\end{array}$ \\
Dormir & 02 & $0.25 \pm 0.70$ & 39 & $4.87 \pm 9.03$ & -1.44 & 0.17 \\
Descansar & 05 & $0.62 \pm 1.76$ & 28 & $3.50 \pm 4.07$ & -1.83 & 0.08 \\
Alimentar-se & 19 & $2.37 \pm 2.19$ & 37 & $4.62 \pm 4.20$ & -1.34 & 0.20 \\
Escalar & 27 & $3.37 \pm 2.66$ & 30 & $3.75 \pm 2.05$ & -0.31 \\
Brincar com outro & 07 & $0.87 \pm 2.47$ & 05 & $0.62 \pm 1.76$ & 0.23 & 0.75 \\
Interagir com outro & 07 & $0.87 \pm 1.45$ & 02 & $0.25 \pm 0.46$ & 1.15 & 0.26 \\
\hline
\end{tabular}

Apesar do macho não ter se mostrado mais ativo com a aplicação dos enriquecimentos, a fêmea teve, conforme já mencionado, um aumento de atividade registrado, inclusive para o comportamento "lamber pata", que é interpretado como uma estereotipia quantitativa (Shepherdson 1998) e já foi observado em tamanduás-bandeira cativos (Braga et al. 2010). Tais 
comportamentos podem estar associados à ausência de estímulos para os animais (Bloomstrand et al. 1986) e, neste caso, a redução na expressão deste comportamento recorrente na fêmea pode ser um indicativo da eficiência dos enriquecimentos aplicados. Vale salientar que o Aquário de São Paulo apresenta programas de aplicação de enriquecimentos regulares, e que o enriquecimento especificamente aplicado aos tamanduás-mirins foi suspenso um mês antes do início deste trabalho a fim de evitar qualquer interferência neste trabalho. A redução nos comportamentos de manutenção da fêmea também foi observada com a aplicação dos enriquecimentos, o que pode indicar maior atenção do animal aos objetos dispostos no recinto não obstante a manipulação direta dos elementos do enriquecimento não tenha sido um comportamento com expressão significativa. A despeito disto, a inserção dos mesmos no recinto teria motivado os animais à maior atividade, promovendo estímulos neste sentido e, portanto, cumprindo o seu papel de exercitar reações diferenciadas frente a tais elementos e evitando a expressão de comportamentos de letargia e inatividade. Uma maior atividade comportamental não estereotipada reflete de fato uma melhor aptidão dos animais para enfrentar eventos de estresse ou minorar os efeitos do tédio (Calrstead 1996), e os enriquecimentos aqui aplicados, logo, contribuíram para aumentar a variabilidade e complexidade do ambiente no qual estes animais são mantidos. Uma análise futura que envolva um $\mathrm{n}$ maior de indivíduos pode embasar de modo mais consistente o que as observações mostraram e o que, de fato, vem sendo mostrado na literatura corrente a respeito da aplicação de enriquecimentos para animais em cativeiro.

\section{Agradecimentos}

Os autores gostariam de agradecer aos funcionários do Aquário de São Paulo que viabilizaram a logística deste trabalho. Aos avaliadores anônimos pela revisão crítica do manuscrito.

\section{Referências}

Altmann J. (1974) Observational study of behavior: Sampling methods. Behaviour, 49: 227-267.

Bloomstrand K.R., Riddle K.A. \& Maple T.L. (1986) Objective evaluation of a behavioral enrichment device for captive chimpanzees (Pan troglodytes). Zoo Biology, 5: 293-300. DOI: 10.1002/zoo.1430050307

Borges M.P., Byk J. \& Del Claro K. (2011) Influência de técnicas de enriquecimento ambiental no aumento do bem-estar de Callithrix penicillata (E. Geoggroy, 1812) (Primates: Callitrichidae). Biotemas, 24: 83-94. DOI: 10.5007/2175-7925.2011v24n1p83

Braga F.G., Santos R.E.F. \& Batista A.C. (2010) Marking behavior of the giant anteater Myrmecophaga tridactyla (Mammalia: Myrmecophagidae) in Southern Brazil. Zoology, 27: 7-12. DOI: $10.1590 /$ S1984-46702010000100002

Carlstead K. (1996) Effects of captivity on the behaviour of wild animals (p. 317-333). In: Kleiman D.G., Allen M.E., Thompson K.V., Lumpkin S. \& Harris H. (Eds). Wild Animals in Captivity: Principles and Techniques. Chicago: The University of Chicago Press. 639 p.

Carducci L.M. \& Jakob E.M. (2000) Rearing environment affects behaviour of jumping spiders. Animal Behaviour, 59: 39-46. DOI: 10.1006/anbe.1999.1282

Catapani M. (2014) Comportamento de tamanduá-mirim, Tamandua tetradactyla (Linnaeus, 1758) (Pilosa, Myrmecophagidae) em condições de cativeiro: implicações ao bem-estar. Dissertação de Mestrado, Programa de Pós-Graduação em Ecologia e Recursos Naturais. Universidade Federal de São Carlos, São Carlos, São Paulo.

Dias E.L., Martins A.C., Pessutti C. \& Barrella W. (2010) Enriquecimento Ambiental no Recinto do Mutum-de-Penacho (Crax fasciolata) do Parque Zoológico Municipal "Quinzinho de Barros", Sorocaba-SP. Revista Eletrônica de Biologia, 3: 20-38.

Eisenberg J.F. \& Redford K.H. (1999) Mammals of the Neotropics: The Central Neotropics. Chicago: University of chicago Press. 624 p.

Hashimoto C.Y. (2008) Comportamento em cativeiro e teste de eficácia de técnicas de enriquecimento ambiental (físico e alimentar) para Jaguatiricas (Leopardus pardalis). 
Dissertação de Mestrado, Programa de Pós-Graduação em Psicologia, Universidade de São Paulo, São Paulo.

Hurme K., Gonzalez K., Halvorsen M., Foster B., Moore D. \& Chepko-Sade B.D. (2003) Environment enrichment for dendrobatid frogs. Journal of Applied Animal Welfare Sciences, 6: 285-299. DOI: 10.1207/s15327604jaws0604_3

IBAMA (2015) Portaria Normativa $\mathrm{n}^{\circ}$ 07. Brasília, $50 \mathrm{p}$.

Lubin Y.D. (1983) Eating ants is no picnic. Natural History, 10: 55-57.

Maple T.L. (2007) Toward a science of welfare for animals in the zoo. Journal of Applied Animal Welfare Sciences, 10(1): 63-70. DOI: 10.1080/10888700701277659

Medri I.M., Mourão G. \& Harada A.Y. (2003) Dieta de Tamanduá-Bandeira (Myrmecophaga tridactyla) no pantanal da Nhecolândia, Brasil. Edentata, 5: 29-34.

Montgomery G.G. (1985) The evolution and ecology of armadillos, sloths and vermilinguas. Washington, D.C.: Smithsonian Institution Press. 451 p.

Redford K.H. (1994) The edentates of the Cerrado. Edentata, 1(1): 4-10.

Rodrigues F.H.G. \& Marinho-Filho J.S. (2003) Home ranges of translocated less anteaters (Tamandua tetradactyla) in the Cerrado of Brazil. Oryx, 35(2): 166-169. DOI: 10.1046/j.13653008.2001.00162.x

Rodrigues F.H.G. \& Marinho-Filho J.S. (2008) Diurnal rest sites of translocated less anteaters (Tamandua tetradactyla) in the Cerrado of Brazil. Edentata, 5: 44-46.

Shepherdson D.J. (1998) Tracing the path of enviromental enrichment in zoos (p. 1-12). In: Shepherdson D.J., Mellen J.D. \& Hutchins M. (Orgs). Second Nature: environmental enrichment for captive animals. Washington, D.C.: Smithsonian Institution Press. 376 p.

Silveira E.K.P. da (1968) Notas sobre a história natural do tamanduá mirim (Tamandua tetradactyla chiriquensis J. A. Allen 1904, Myrmecophagidae), com referências à fauna do istmo do Panamá. Vellozia, 6: 9-31.

Superina M. (2012) Um Passeio pela biologia dos tamanduás (p. 26-37). In: Miranda F.R. (Ed.). Manutenção de tamanduás em cativeiro. São Carlos: Editora Cubo. 302 p.

Viscaíno S.F. \& Loughry W.J. (2008) The Biology of the Xenarthra. Gainesville: University Press of Florida. 370 p. 